\title{
OPEN Synthesis, characterization and photothermal analysis of nanostructured hydrides of Pd and $\mathrm{PdCeO}_{2}$
}

Cláudia C. R. Cruz ${ }^{1}$, Nilton P. da Silva ${ }^{2,3}$, Amanda V. Castilho $^{1}$, Viviane A. Favre-Nicolin ${ }^{4}$, Claudio L. Cesar ${ }^{5}$, Helcio R. B. Orlande ${ }^{1,2}$ \& Dilson S. Dos Santos ${ }^{1,6 \bowtie}$

Hyperthermia was shown to be an important co-adjuvant therapy to conventional cancer treatments. Nanoparticles can be used in the hyperthermia therapy to improve the localized absorption of energy imposed by external sources, in order to kill tumor cells solely by the effect of heat and with minimum thermal damage to surrounding healthy cells. Nanoparticles can also serve as carriers of drugs that specifically act on the tumor when heated, including hydrogen that can be desorbed to locally promote an antioxidant effect and reduce the viability of cancer cells. In this context, palladium hydride nanoparticles emerge as promising materials for the hyperthermia therapy. In this study, palladium nanocubes (PdNC) and $\mathrm{PdCeO}_{2}$ nanoparticles were synthesized. Nanofluids produced with these nanomaterials were hydrogenated and then tested to examine their photothermal effects. Nanofluids made of $\mathrm{PdH}_{\mathrm{x}}$ nanoparticles presented significant temperature increases of more than $30^{\circ} \mathrm{C}$ under 3 min of diode-laser irradiation. On the other hand, nanofluids with $\mathrm{PdCeO}_{2} \mathrm{H}$ nanoparticles presented temperature increases around $11^{\circ} \mathrm{C}$ under the same experimental conditions. The behavior observed with the $\mathrm{PdCeO}_{2} \mathrm{H}$ nanofluids can be attributed to the effect of $\mathrm{H}^{+}$in reducing $\mathrm{Ce}^{+4}$ to $\mathrm{Ce}^{+3}$.

Nanoparticles of noble metals and ordinary metallic alloys have been successfully used in the hyperthermia cancer therapy ${ }^{1}$. Nanoparticles naturally tend to concentrate in the tumor, thus locally increasing the absorption of external energy sources imposed during the hyperthermia therapy. As a result, thermal damage can be mainly imposed to the tumor cells, without significant effects on the surrounding healthy cells ${ }^{2}$. The use of hydrogen is very promising for the hyperthermia cancer therapy, due to its important role in physiological regulation, besides exhibiting good biomedical applicability and biosafety ${ }^{3}$. Hydrogen is a therapeutic antioxidant that can selectively reduce the hydroxyl radical $\left(\mathrm{OH}^{-}\right)$, the most cytotoxic of the reactive oxygen species produced by tumor cells ${ }^{4}$. For the hyperthermia therapy, hydrogen must be safely and effectively stored in nanoparticles to target specific cancer cells and allow its controlled release. Nanoparticles of palladium hydride can store large amounts of hydrogen and exhibit good biocompatibility, as well as high photothermal conversion efficiencies in the near infrared range $e^{5,6}$.

Palladium and its alloys have been widely used in various engineering applications, mainly as nanocrystalline powders, especially as palladium nanocubes ${ }^{7-13}$. Different uses for this material have been reported, such as in catalysis ${ }^{14}$, sensors ${ }^{15}$, waste treatment ${ }^{16}$ and hydrogen storage ${ }^{17,18}$, in addition to cancer treatment ${ }^{19,20}$. The ability of palladium to absorb large amounts of hydrogen at room temperature and atmospheric pressure, thus forming a palladium hydride $\left(\mathrm{PdH}_{\mathrm{x}}\right)$, is one of its main advantages ${ }^{21}$. Both $\mathrm{Pd}$ and $\mathrm{PdH}_{\mathrm{x}}$ have a fcc structure, but with different lattice parameters of $3.88 \AA$ and $4.10 \AA$, respectively. Hydrogen occupies the octahedral sites of the fcc structure and diffuses through pure annealed Pd with a room temperature diffusivity of about $10^{-11} \mathrm{~m}^{2} \mathrm{~s}^{-122}$. Palladium hydride decomposition is slower than its formation at room temperature ${ }^{23}$.

\footnotetext{
${ }^{1}$ Program of Nanotechnology Engineering, COPPE, Federal University of Rio de Janeiro, Rio de Janeiro, RJ, Brazil. ${ }^{2}$ Program of Mechanical Engineering, COPPE - Oncobiology Program, Federal University of Rio de Janeiro, Rio de Janeiro, RJ, Brazil. ${ }^{3}$ Department of Mechanical Engineering, Federal University of Amazonas - UFAM, Manaus, AM, Brazil. ${ }^{4}$ Federal Institute of Education, Science and Technology of Espírito Santo - IFES, Vitoria, ES, Brazil. ${ }^{5}$ Institute of Physics, Federal University of Rio de Janeiro, Rio de Janeiro, RJ, Brazil. ${ }^{6}$ Program of Metallurgical and Materials Engineering, COPPE, Federal University of Rio de Janeiro, Rio de Janeiro, RJ, Brazil. ${ }^{凶}$ email: dilson@metalmat.ufrj.br
} 

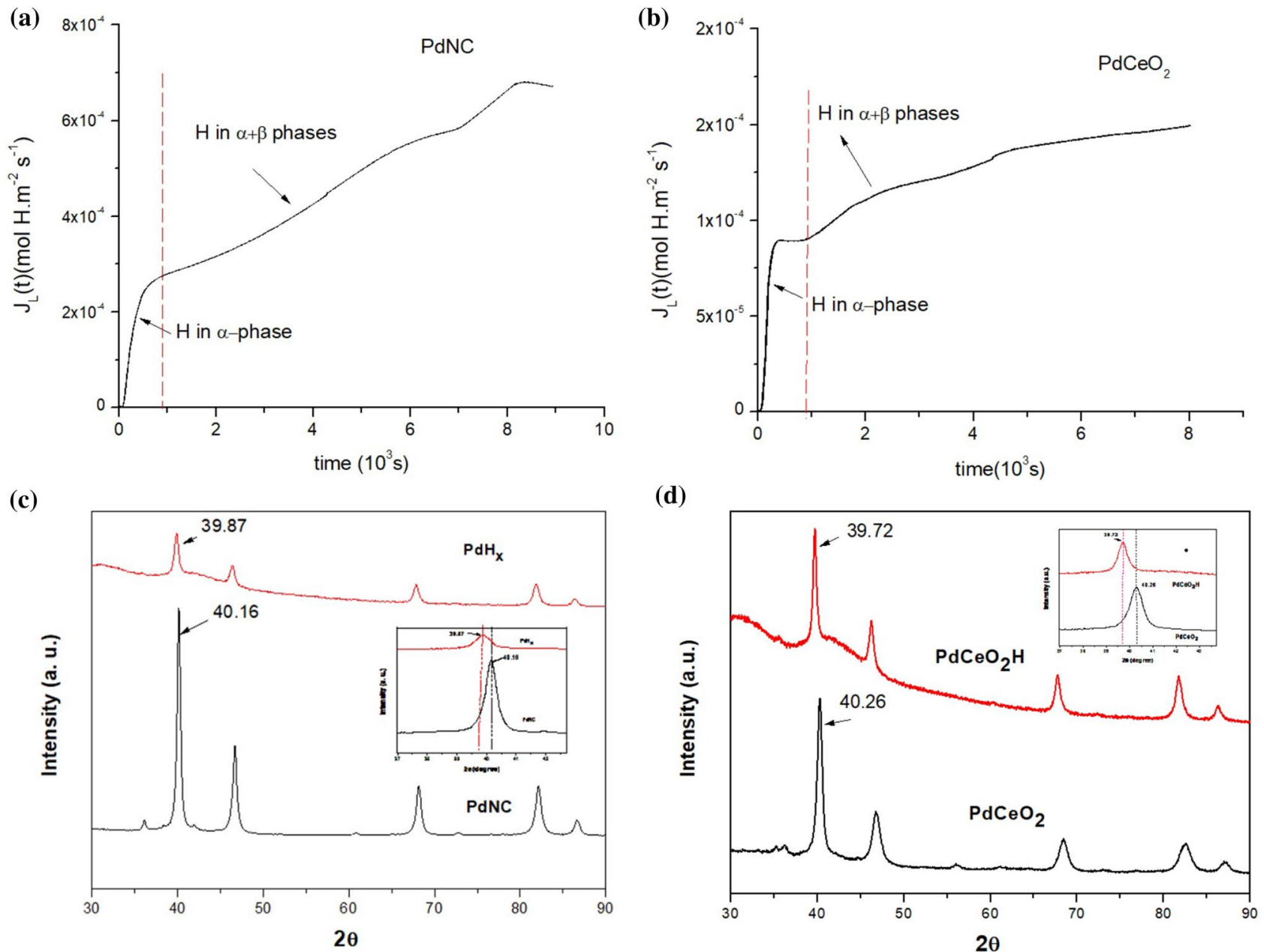

Figure 1. Hydrogen permeation curves obtained with current density of $5 \mathrm{~mA} / \mathrm{cm}^{2}$ for pure $\mathrm{Pd}(\mathbf{a})$ and $\mathrm{PdCeO}_{2}$ internally oxidized (b). XRD pattern for $\mathrm{PdNC}(\mathbf{c})$ and $\mathrm{PdCeO}_{2}(\mathbf{d})$ before and after hydrogenation.

When compared to their bulk versions, nanoscale metallic hydrides present faster hydrogen absorption and release reaction kinetics, due to their larger surface area and lower desorption temperature and activation energies. Thus, nanocrystalline palladium reacts fast for the formation of palladium hydride $\left(\mathrm{PdH}_{\mathrm{x}}\right)$. It can also release hydrogen and regenerate the metal at a high rate, as well as be recharged afterwards ${ }^{24}$.

Palladium nanocubes and nanoparticles of $\mathrm{PdCeO}_{2}$ were manufactured in this work. The hydrogen diffusivity of $\mathrm{PdCeO}_{2}$ is smaller than those of pure palladium in crystalline or nanocube forms. This is due to the hydrogen trap effect of $\mathrm{CeO}_{2}$ in the Pd matrix, which delays the hydrogen desorption. Therefore, nanoparticles made of $\mathrm{PdCeO}_{2}$ can potentially increase the period between production and its practical application, such as in the hyperthermia treatment. This material is also non-toxic, presents good bio-affinity and is a potent antioxidant for reactive oxygen species (ROS) $)^{25,26}$. Azambuja et al. ${ }^{27}$ performed the synthesis of nanostructured Pd soaked with $\mathrm{CeO}_{2}$ in its matrix through internal oxidation.

We note, however, that the use of palladium nanoparticles to treat diseases still has its limitations. This is due to the fact that the produced nanoparticles may contain materials that are not biocompatible, like metallic powders, salts (acetate, chloride) and other contaminants derived from the synthesis ${ }^{28}$.

The present work aims at synthesizing and characterizing palladium nanocubes (PdNC) and the nanostructured $\mathrm{PdCeO}_{2}$ alloy. It also aims at evaluating the photothermal effects of nanofluids obtained with the dispersion of these nanoparticles in distilled water. Some of the nanofluids were bubbled with hydrogen in order to form hydrides of Pd nanocubes and $\mathrm{PdCeO}_{2}$ nanoparticles. Nanofluids with different concentrations were continuously heated with a diode-laser $(829.1 \mathrm{~nm})$ during experiments with controlled powers of $154 \mathrm{~mW}, 218 \mathrm{~mW}$ and $439 \mathrm{~mW}$.

\section{Results and discussion}

Figure 1a,b show the hydrogen permeation curves for pure $\mathrm{Pd}$ and $\mathrm{PdCeO}_{2}$, respectively, obtained at room temperature under a current density of $5 \mathrm{~mA} / \mathrm{cm}^{2}$. These figures provide the hydrogen diffusivity of $\mathrm{Pd}$ as $3.3 \times 10^{-11} \mathrm{~m}^{2} \mathrm{~s}^{-1}$ (Fig. 1a), which is almost six times larger than that of $\mathrm{PdCeO}_{2}\left(0.6 \times 10^{-12} \mathrm{~m}^{2} \mathrm{~s}^{-1}\right.$-see Fig. 1b). Thus, $\mathrm{PdCeO}_{2}$ absorbs less hydrogen during the hydrogenation period. The hydrogen diffusivity decreases with the addition of $\mathrm{CeO}_{2}$ in $\mathrm{Pd}$, due to the accommodation of this oxide in the Pd matrix. The Ce-oxide lattice has 


\begin{tabular}{|l|l|}
\hline Material & Lattice parameters $(\AA)$ \\
\hline $\mathrm{PdNC}$ & 3.89 \\
\hline $\mathrm{PdH}_{\mathrm{x}}$ & 3.92 \\
\hline $\mathrm{PdCeO}_{2}$ & 3.88 \\
\hline $\mathrm{PdCeO}_{2} \mathrm{H}$ & 3.91 \\
\hline
\end{tabular}

Table 1. Lattice parameters for $\mathrm{PdNC}$ and $\mathrm{PdCeO}_{2}$ before and after hydrogenation.

a coincidence with a direction [111] of the Pd lattice. In this case, the interface between $\mathrm{CeO}_{2}$ and Pd is semicoherent and can effectively trap hydrogen atoms. Our objective with the addition of $\mathrm{CeO}_{2}$ in $\mathrm{Pd}$ was to reduce the hydrogen diffusivity using a biocompatible material, in order to allow longer time periods between production and use of the hydrides. Besides that, its valence also plays an important role in relation to the hydrogenation, as discussed below.

Figure $1 \mathrm{c}, \mathrm{d}$ show the X-ray diffraction patterns obtained for pure $\mathrm{Pd}$ and $\mathrm{PdCeO}_{2}$, respectively, before and after hydrogenation. The displacement of the peaks to the left, which represents a significant absorption of hydrogen, can be observed in Fig. 1c,d. The intensity of the diffraction peaks in the hydrogenated $\mathrm{PdCeO}_{2}$ alloy is lower than in the original material. Hydride precipitation, when it occurs, decreases the diffraction intensity of the primary phase. Although the hydride peaks are not clear, the shift to the left and the broadening of $\mathrm{Pd}$ peaks reveal the beginning of the phase transformation.

From the diffraction patterns of Fig. 1c,d it was possible to determine with Bragg's Law the lattice parameters that are presented in Table 1. These lattice parameters reveal that the amount of hydrogen absorbed was $0.15 \%$ $\mathrm{H} / \mathrm{Pd}$ for PdNC and $0.11 \% \mathrm{H} / \mathrm{Pd}$ for $\mathrm{PdCeO}_{2}$. Hydrogen at low concentrations is sufficient to form hydrides but does not necessarily form the extra peaks related to the reflection of the $\mathrm{PdH}_{\mathrm{x}}$ phase. These results obtained here are in accordance with those available in the literature ${ }^{3,29}$.

Figure $2 \mathrm{a}-\mathrm{f}$ show the microstructural analysis for both materials obtained via SEM and TEM, respectively. The formation of Pd nanocubes with mean size $20 \mathrm{~nm}$ can be observed by SEM (Fig. 2a) and by TEM (Fig. 2b,c). The nano Ce oxides precipitated in the Pd matrix present nanospheres and large needles ${ }^{27}$. After milling, only these nanoprecipitates were observed. The $\mathrm{CeO}_{2}$ was always incorporated within the Pd matrix (Fig. 2e,f).

The use of $\mathrm{CTAB}$ in the synthesis of the nanocubes is important to stabilize the cubic morphology in the presence of bromide ${ }^{30}$. In addition, in a synthesis starting from $\mathrm{Na}_{2} \mathrm{PdCl}_{4}$ the CTAB has another important role, by reacting with the precursor and forming a complex as shown by reaction I. The addition of an excess of ascorbic acid to the colloidal complex reduces the dissociative organic salt and shifts the reaction balance slowly toward dissolution (reaction II) ${ }^{31}$. After mixing CTAB with $\mathrm{Na}_{2} \mathrm{PdCl}_{4}$, the bromide ions $\left(\mathrm{Br}^{-}\right)$dissociated from the surfactant can replace the chloride ions $\left(\mathrm{Cl}^{-}\right)$and bind to $\mathrm{Pd}^{2+}$, which then reacts with $\mathrm{CTAB}^{+}$to form an organic salt (reaction I).

$$
\begin{gathered}
{\left[\mathrm{PdCl}_{4-n} \mathrm{Br}_{n}\right]_{(a q)}^{-2}+2 \mathrm{CTA}_{(a q)}^{+} \leftrightarrow(\mathrm{CTA})_{2}\left[\mathrm{PdCl}_{4-n} \mathrm{Br}_{n}\right]_{(s)}} \\
{\left[\mathrm{PdCl}_{4-n} \mathrm{Br}_{n}\right]_{(a q)}^{-2}+\mathrm{C}_{6} \mathrm{H}_{6} \mathrm{O}_{4}(\mathrm{OH})_{2(a q)} \rightarrow \mathrm{Pd}(\mathrm{NPs})+\mathrm{C}_{6} \mathrm{H}_{6} \mathrm{O}_{6(a q)}+(4-n) \mathrm{Cl}_{(a q)}^{-}+2 \mathrm{H}_{(a q)}^{+}+\mathrm{nBr}_{(a q)}^{-}}
\end{gathered}
$$

Figure $2 \mathrm{~g}$ shows the EDS mapping of Pd nanocubes, where the presence of only palladium can be observed, free of contaminants from the organic reagents used in the reaction. Figure $2 \mathrm{~g}$ shows the presence of clusters of Pd nanocubes. Under higher magnification, the cubic morphology becomes well defined. Figure $2 \mathrm{~h}$ shows a $\mathrm{CeO}_{2}$ particle bound to $\mathrm{Pd}$. In the nanostructured $\mathrm{PdCeO}_{2}$ alloy, the presence of iron due to the milling process was observed. Although being undesirable in the alloy, Fe would not be a concern for the hyperthermia cancer treatment application of the nanocubes, because it is a biocompatible element.

Zhao et al. ${ }^{3}$ presented the absorption spectrum of the palladium nanocubes and their respective hydrides up to a concentration of $0.06 \mathrm{~g} / \mathrm{L}$, in the UV-VIS-NIR, showing that the palladium nanocubes absorb more strongly in the ultra-violet (UV) range. In the presence of hydrogen, there is an increase in the absorption curve in the visible and near infrared (VIS-NIR) ranges, starting from $500 \mathrm{~nm}$. Thus, the nanostructured palladium hydride has great potential for the hyperthermia therapy, since it has a large absorptivity in the NIR range and allows a controlled release of hydrogen.

In order to evaluate the photothermal effects of the nanoparticles produced in this work, distilled water nanofluids were prepared and tested under diode-laser heating, as described in the next section. Figure 3a-c present the temperature variations observed with the PdNC nanofluid and its hydrogenated version for two concentrations $(0.2$ and $0.4 \mathrm{~g} / \mathrm{L})$ and the powers of $154 \mathrm{~mW}, 218 \mathrm{~mW}$ and $439 \mathrm{~mW}$, respectively. Similarly, the temperature variations observed with the $\mathrm{PdCeO}_{2}$ nanofluid $(0.4 \mathrm{~g} / \mathrm{L})$ and its hydrogenated version are presented in Fig. $4 \mathrm{a}-\mathrm{c}$ for the same diode-laser powers, respectively. The temperature variations presented in these figures were measured with an infrared camera and correspond to the pixel at the center of the fluid surface. The curves presented in Figs. 3 and 4 include the corresponding measurement uncertainties.

Figure $3 \mathrm{a}-\mathrm{c}$ show that the nanofluids made with Pd hydride nanocubes exhibited much larger temperature variations than the nanofluids made with Pd nanocubes. This was expected due to the thermal effect of hydrogen during desorption. Under the laser powers of $154 \mathrm{~mW}$ and $218 \mathrm{~mW}$, the presence of hydrogen caused larger temperature variations in the nanofluids of low concentration (e.g., Pd hydride nanocubes with concentration 

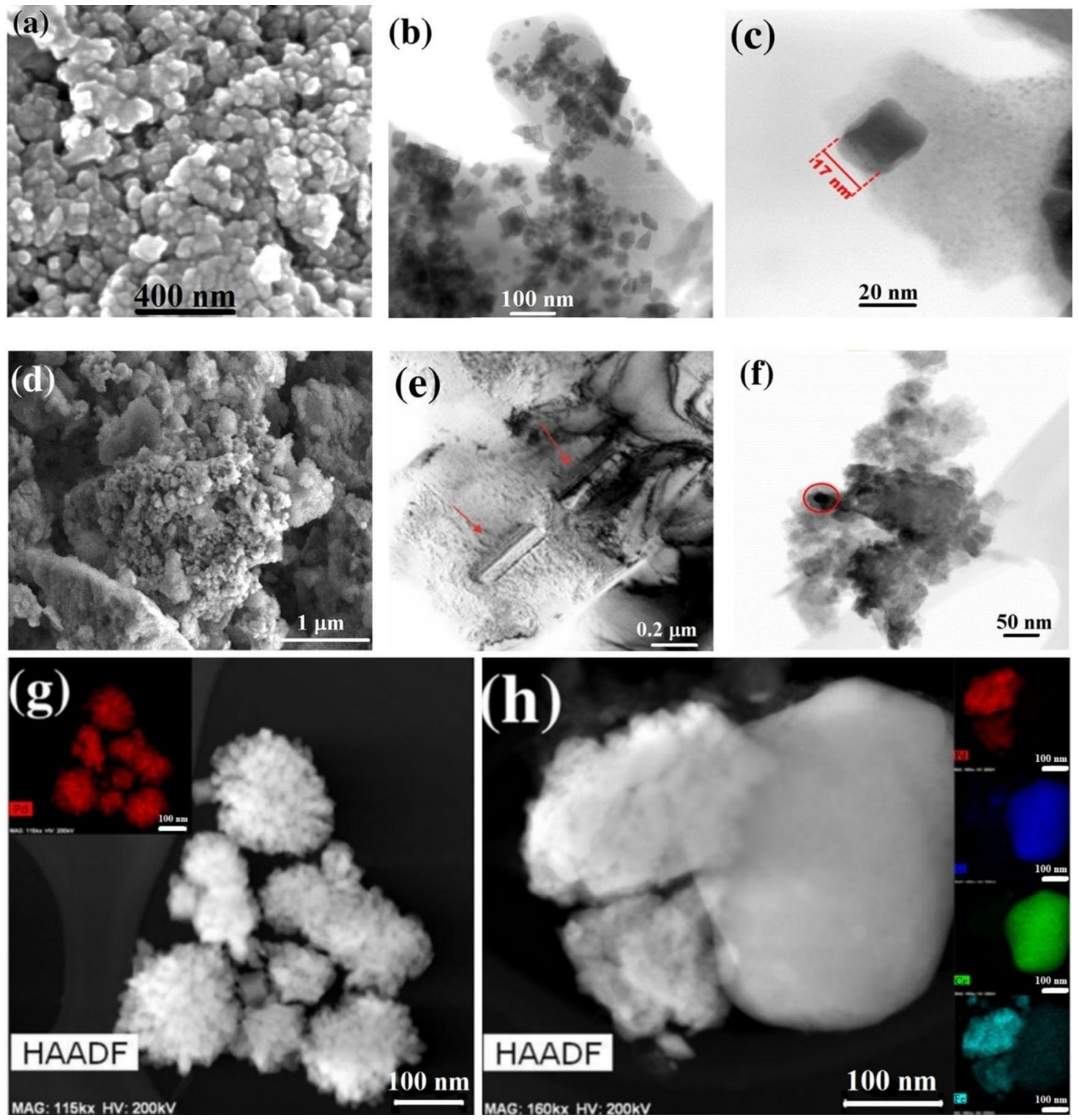

Figure 2. (a) SEM image of Pd nanocubes. (b) TEM image of Pd nanocubes. (c) TEM image of a Pd nanocube showing well-faceted interfaces. (d) SEM image of $\mathrm{PdCeO}_{2}$ alloy. (e) TEM image of $\mathrm{PdCeO}_{2}$ alloy before mechanical milling. (f) TEM image of $\mathrm{PdCeO}_{2}$ alloy after mechanical milling. (g) EDS mapping of Pd nanocubes. (h) EDS mapping of $\mathrm{PdCeO}_{2}$ nanoparticles. In the spotlight: $\mathrm{Pd}$ (red), O (blue), $\mathrm{Ce}$ (green) and $\mathrm{Fe}$ (light blue).

of $0.2 \mathrm{~g} / \mathrm{L}$ under $154 \mathrm{~mW}$ heating presented maximum $\Delta T=9^{\circ} \mathrm{C}$ ) than those presented by the nanofluids of $\mathrm{Pd}$ nanocubes of high concentration (Pd nanocubes with concentration of $0.4 \mathrm{~g} / \mathrm{L}$ under $154 \mathrm{~mW}$ presented maximum $\Delta T=8^{\circ} \mathrm{C}$ ). This demonstrates that the effect of hydrogen desorption on the temperature variation is predominant over that of the absorption cross section resulting from the nanoparticles in the fluid. At the highest power of $439 \mathrm{~mW}$, the concentration of nanoparticles has a more significant effect; the larger concentrations resulted in larger temperature variations, regardless the nanoparticles $\left(\mathrm{PdNC}\right.$ or $\left.\mathrm{PdH}_{\mathrm{x}}\right)$ in the nanofluids. Therefore, for larger concentrations the effects of the larger absorption cross sections of the nanofluids are more important for the temperature increase than the effects of the hydrogen desorption. In addition, the curves for the nanofluids of Pd hydride nanocubes exhibited different profiles, presenting a steeper temperature variation at small times due to the presence of hydrogen, followed by profiles similar to those of the nanofluids of PdNC.

Figure $4 \mathrm{a}-\mathrm{c}$ show the measured temperature variations of $\mathrm{PdCeO}_{2}$ and $\mathrm{PdCeO}_{2} \mathrm{H}$ nanofluids with a concentration of $0.4 \mathrm{~g} / \mathrm{L}$, obtained with a heating duration of $3 \mathrm{~min}$ and laser powers of $154 \mathrm{~mW}, 218 \mathrm{~mW}$ and 439 $\mathrm{mW}$, respectively.

Table 2 summarizes the temperature variations of the nanofluids after three minutes of diode-laser heating, as shown by Figs. 3 and 4. A comparison of these temperature variations reveals that Pd nanocubes absorb less energy in the NIR than $\mathrm{PdCeO}_{2}$ nanoparticles. The maximum temperature variation obtained for PdNC was $25.3^{\circ} \mathrm{C}$, while for $\mathrm{PdCeO}_{2}$ it was $27.1^{\circ} \mathrm{C}$, for the same power of $439 \mathrm{~mW}$ and same concentration of $0.4 \mathrm{~g} / \mathrm{L}$. The 

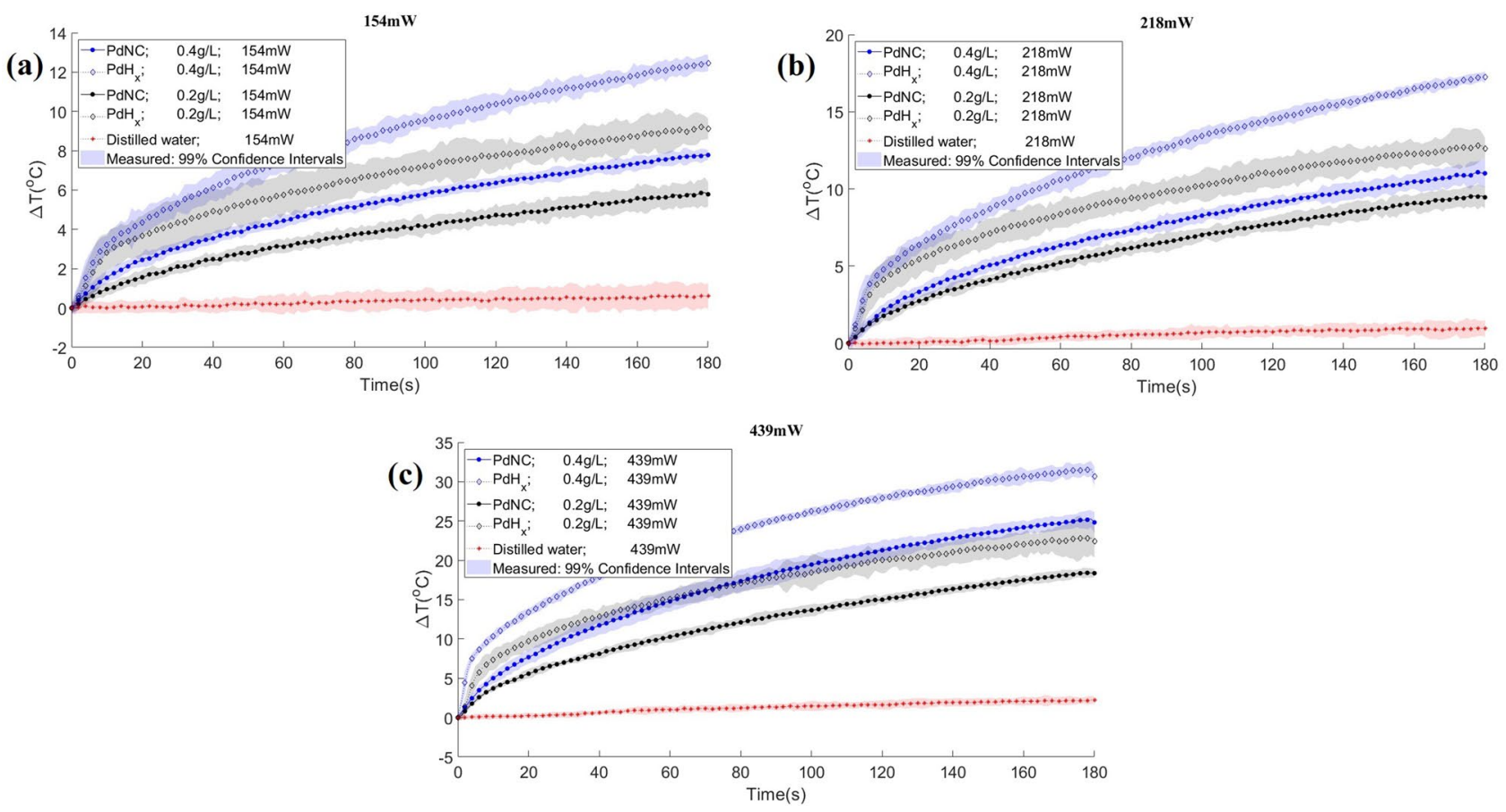

Figure 3. Experimental temperature variations of nanofluids made of Pd nanocubes and Pd hydride nanocubes, with concentrations of $0.2 \mathrm{~g} / \mathrm{L}$ (black) and $0.4 \mathrm{~g} / \mathrm{L}$ (blue). Heating performed during $3 \mathrm{~min}$ with three powers: (a) $154 \mathrm{~mW}$, (b) $218 \mathrm{~mW}$ and (c) $439 \mathrm{~mW}$. Distilled water (red) was used as white.
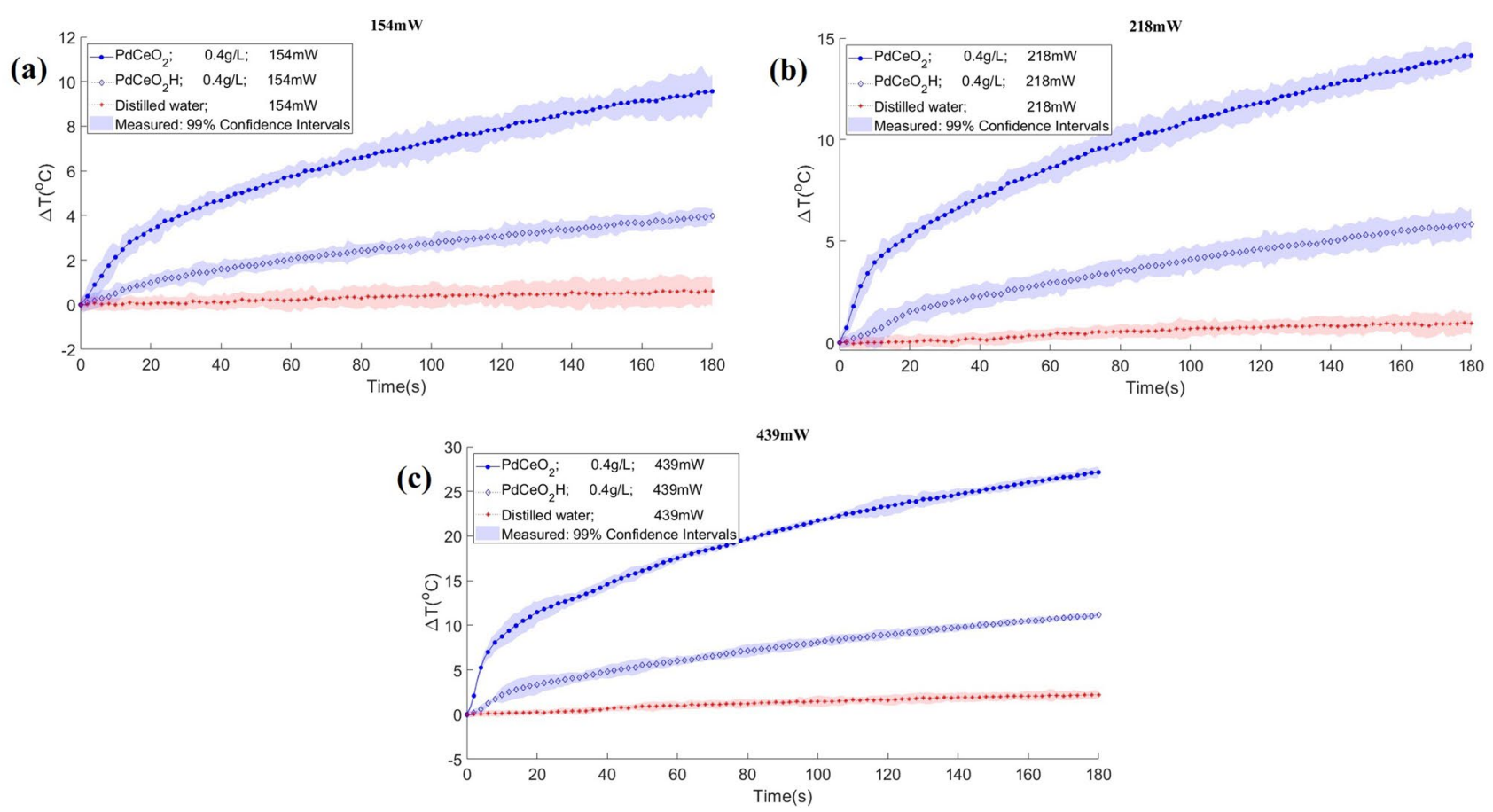

Figure 4. Experimental temperature variation of nano $\mathrm{Pd}$ cerium oxide, $\mathrm{PdCeO}_{2}$ (filled circle) and nano $\mathrm{Pd}$ hydride cerium oxide, $\mathrm{PdCeO}_{2} \mathrm{H}$ (filled diamond) nanofluids with a concentration of $0.4 \mathrm{~g} / \mathrm{L}$. Heating performed during $3 \mathrm{~min}$ with three powers: (a) $154 \mathrm{~mW}$, (b) $218 \mathrm{~mW}$ and (c) $439 \mathrm{~mW}$. Distilled water (red) was used as white. 


\begin{tabular}{|c|c|c|c|c|c|c|}
\hline \multirow[b]{2}{*}{ Laser power (mW) } & \multicolumn{6}{|c|}{$\Delta \mathrm{T}\left({ }^{\circ} \mathrm{C}\right)$ after $180 \mathrm{~s}$} \\
\hline & PdNC $0.2 \mathrm{~g} / \mathrm{L}$ & $\mathrm{PdH}_{\mathrm{x}} 0.2 \mathrm{~g} / \mathrm{L}$ & PdNC $0.4 \mathrm{~g} / \mathrm{L}$ & $\mathrm{PdH}_{x} 0.4 \mathrm{~g} / \mathrm{L}$ & $\mathrm{PdCeO}_{2} 0.4 \mathrm{~g} / \mathrm{L}$ & $\mathrm{PdCeO}_{2} \mathrm{H} 0.4 \mathrm{~g} / \mathrm{L}$ \\
\hline 154 & 5.9 & 9.2 & 7.8 & 12.5 & 9.6 & 4.0 \\
\hline 218 & 9.5 & 12.8 & 11.1 & 17.3 & 14.1 & 5.8 \\
\hline 439 & 18.4 & 22.8 & 25.3 & 31.7 & 27.1 & 11.2 \\
\hline
\end{tabular}

Table 2. Temperature variations of nanofluids of $\mathrm{PdNC}, \mathrm{PdCeO}_{2}$ and their hydrogenated forms.
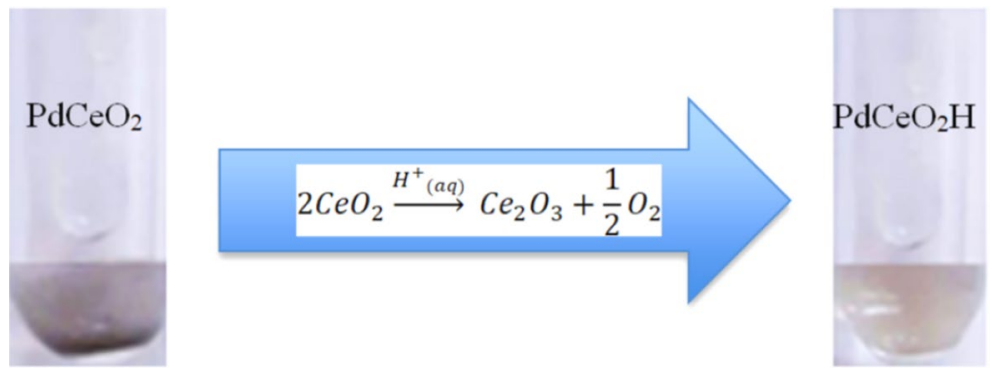

Figure 5. Modification of the color the $\mathrm{PdCeO}_{2}$ nanofluid after hydrogenation.

\begin{tabular}{|c|c|c|c|c|c|}
\hline \multicolumn{6}{|c|}{ Photothermal conversion efficiency (standard deviation), \% } \\
\hline PdNC $0.2 \mathrm{~g} / \mathrm{L}$ & $\mathrm{PdH}_{\mathrm{x}} 0.2 \mathrm{~g} / \mathrm{L}$ & PdNC 0.4 g/L & $\mathrm{PdH}_{\mathrm{x}} 0.4 \mathrm{~g} / \mathrm{L}$ & $\mathrm{PdCeO}_{2} 0.4 \mathrm{~g} / \mathrm{L}$ & $\mathrm{PdCeO}_{2} \mathrm{H} 0.4 \mathrm{~g} / \mathrm{L}$ \\
\hline $42(5)$ & $55(4)$ & $56(4)$ & $70(5)$ & $59(4)$ & $24(4)$ \\
\hline
\end{tabular}

Table 3. Photothermal conversion efficiencies of nanofluids of $\mathrm{Pd}, \mathrm{PdCeO}_{2}$ and their hydrogenated forms with the laser power of $439 \mathrm{~mW}$. Numbers inside parentheses are the standard deviations of the efficiencies.

effect of hydrogen in the nanoparticles was remarkable with PdNC, which promoted a temperature variation of $31.7^{\circ} \mathrm{C}$ at the final time for the largest power and largest concentration examined, while the regular PdNC nanofluid exhibited a temperature variation of $25.3^{\circ} \mathrm{C}$ for the same conditions. On the other hand, Table 2 shows that the presence of hydrogen in the nanofluids of $\mathrm{PdCeO}_{2}$ had the opposite effect. A reduction in the energy absorbed by the nanofluid can be noticed, which reduced the temperature variation from $27.1^{\circ} \mathrm{C}$ for the $\mathrm{PdCeO}_{2}$ nanofluid to $11.2^{\circ} \mathrm{C}$ for the $\mathrm{PdCeO}_{2}$ hydrogenated nanofluid. This result might have been caused by the agglomeration of the nanoparticles after hydrogenation, in addition to possible changes in valence for cerium from $\mathrm{Ce}^{+4}$ to $\mathrm{Ce}^{+3}$. In fact, there was a modification of the color of the $\mathrm{PdCeO}_{2}$ nanofluid when it was hydrogenated, as shown by Fig. 5 .

As an oxide, the most stable form of cerium is $\mathrm{Ce}^{+4}$, where oxygen atoms occupy the tetrahedral positions and a portion of $\mathrm{Ce}^{+3}$ has the positive charge deficiency compensated by oxygen gaps ${ }^{25}$. The concentration of $\mathrm{Ce}^{+3}$ increases with the decrease in particle size ${ }^{25}$ and cerium oxide nanoparticles exhibit a significant amount of this ion. Therefore, the reacting medium will be the determining factor for the oxidation or reduction effects of cerium oxide nanoparticles. The excess of hydrogen causes $\mathrm{Ce}^{+4}$ to react with the hydrogen ions that were dissociated at the palladium surface, thus forming $\mathrm{H}^{+}$that reduces $\mathrm{Ce}^{+4}$ to $\mathrm{Ce}^{+3}$ (reaction III) ${ }^{32}$. This fact modifies the absorption of the material in the NIR, resulting in a lower temperature increase than that of its non-hydrogenated form.

$$
2 \mathrm{CeO}_{2} \stackrel{\mathrm{H}_{(a q)}^{+}}{\longrightarrow} \mathrm{Ce}_{2} \mathrm{O}_{3}+\frac{1}{2} \mathrm{O}_{2}
$$

Das et al. ${ }^{32}$ demonstrated with XPS that $\mathrm{Ce}^{+4}$ and $\mathrm{Ce}^{+3}$ coexist in cerium oxide nanoparticles and that the presence of these two valence states on the nano-Ceria surface act as an antioxidant. Nanoparticles then eliminate the free radicals from the culture system, which gives the cerium oxide nanoparticles unique properties for biomedical use. In addition, they revealed that nano-Ceria exhibit auto-catalytic or cyclic regeneration, that is, in the presence of $\mathrm{H}_{2} \mathrm{O}_{2}$ it is possible to regenerate the $\mathrm{Ce}^{+3} \rightarrow \mathrm{Ce}^{+4} \rightarrow \mathrm{Ce}^{+3}$ system. Therefore, this material has great potential for biological applications with antioxidant activity and pseudo-infinite half-life. Hydrogenation induces the change from $\mathrm{Ce}^{+4}$ to $\mathrm{Ce}^{+3}$ by increasing the hydrogen volume ${ }^{33}$ and modifying material properties.

The photothermal conversion efficiencies of the nanofluids produced in this work are presented in Table 3. These values were obtained with the solution of an inverse problem by using the transient temperature variations with the laser power of $439 \mathrm{~mW}$. These efficiencies follow the same trend of the temperature variations (see also Table 2). For PdNC, the efficiencies increased with the hydrogenation of the nanofluids, but the opposite behavior was observed with $\mathrm{PdCeO}_{2}$ nanoparticles. Also, the efficiencies increased with the concentration of PdNC. The maximum efficiency (70\%) was obtained with the hydrogenated PdNC nanofluid with concentration of $0.4 \mathrm{~g} / \mathrm{L}$ 


\section{MEASURED TEMPERATURES}

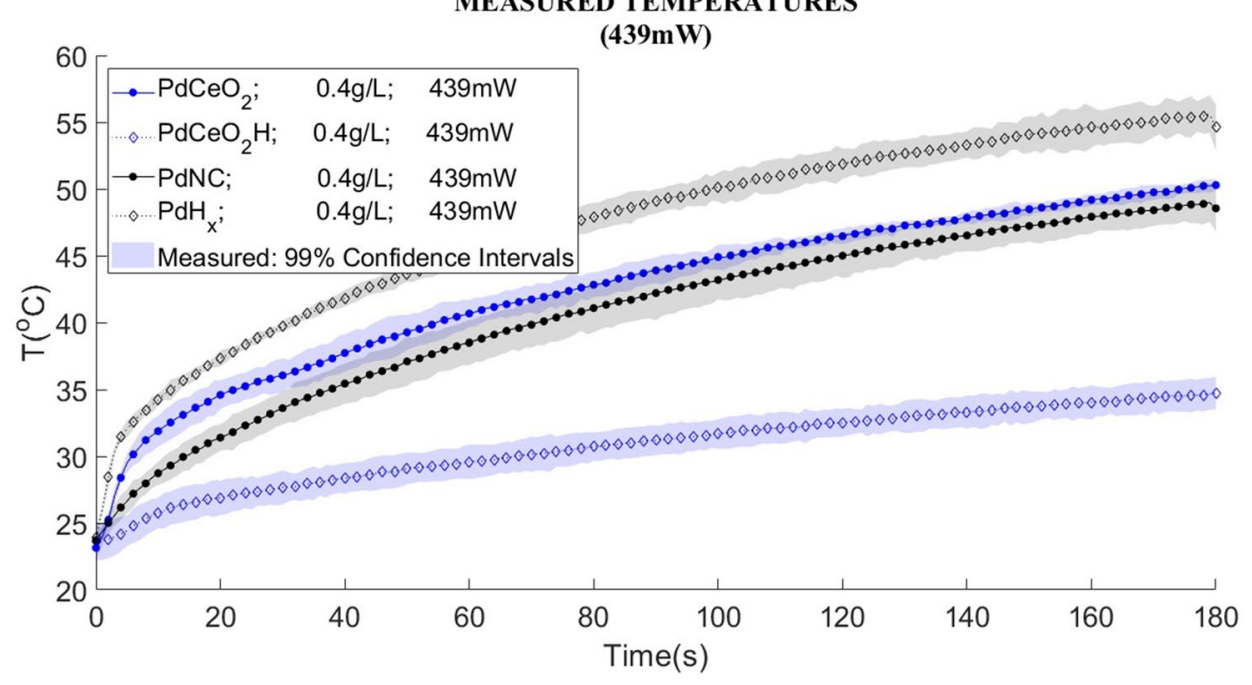

Figure 6. Temperature for palladium and $\mathrm{PdCeO}_{2}$ nanofluids.

of $\mathrm{PdH}_{\mathrm{x}}$, while the minimum efficiency (24\%) was observed with the $\mathrm{PdCeO}_{2} \mathrm{H}$ nanofluid. The values reported in Table 3 for PdNC nanofluids are in accordance with those reported in the literature ${ }^{3}$. The photothermal conversion efficiencies obtained with the other laser powers were similar to those presented in Table 3, but exhibited larger standard deviations; they are not reported here for the sake of brevity.

Figure 6 summarizes the temperature variations for nanofluids of $\mathrm{PdNC}, \mathrm{PdCeO}_{2}$ and their hydrogenated forms, obtained with the laser heating power of $439 \mathrm{~mW}$. Although the absorption of the diode-laser energy in the hydrogenated $\mathrm{PdCeO}_{2}$ nanofluid was smaller than for the other nanofluids (Table 3 and Fig. 6), the resulting temperature increase might still be appropriate for the hyperthermia treatment of cancer depending on the aimed application, which may involve mild temperature increases to make the tumor cells more susceptible to chemotherapy or radiotherapy treatments.

The results obtained in the present work demonstrate the immense potential of Pd nanocubes and nanoparticles of $\mathrm{PdCeO}_{2}$, as well as their hydrogenated forms, in applications such as the hyperthermia therapy of cancer.

\section{Methods}

Hydrogen permeation tests were performed at room temperature with foils about $150 \mu \mathrm{m}$ thick, of pure palladium and $\mathrm{PdCeO}_{2}$. A cathodic current density of $5 \mathrm{~mA} \mathrm{~cm}^{-2}$, which was enough to produce hydride on the surface of the cathodic loading side, was applied to each material. In order to calculate the apparent hydrogen diffusion coefficient, electrochemical permeation tests were performed using a double-cell experiment separated by the sample ${ }^{34}$. An oxidation cell (detection side) was filled with $\mathrm{NaOH} 0.1 \mathrm{M}$ and the electrochemical potential used was obtained from open potentiometric circuit. A reduction cell (cathodic charging side) was filled with $0.1 \mathrm{M}$ $\mathrm{H}_{2} \mathrm{SO}_{4}+2 \mathrm{mg} / \mathrm{l} \mathrm{As}_{2} \mathrm{O}_{3}$ solution. The anodic current was detected on the cell oxidation side. Both currents were generated or detected by an AUTOLAB PGSTAT100N potentiostat.

The apparent hydrogen diffusion coefficient $\left(D_{a p p}\right)$ was calculated according to ${ }^{18}$ :

$$
D_{a p p}=0.76 \frac{L^{2}}{\pi^{2} t_{b}}
$$

where $D_{a p p}$ is the apparent diffusion coefficient, $L$ is the thickness of the sample and $t_{b}$ is the breakthrough time, which corresponds to the time when the first hydrogen atoms permeating through the sample are detected.

For single-phase samples without hydride formation, the permeation curve is sigmoidal. When hydride reaction takes place, the hydrogen permeation curves exhibit a different evolution, which corresponds to hydride nucleation and growth during the test.

Palladium nanocubes (PdNC) were prepared through a precipitation technique using $10 \mathrm{mM}$ aqueous of $\mathrm{Na}_{2} \mathrm{PdCl}_{4}$ (disodium tetrachloropalladate, Sigma-Aldrich 98\%) and CTAB (cetyltrimethylammonium bromide, Sigma-Aldrich 98\%) added to a solution 0.1 M of ascorbic acid (Sigma-Aldrich 99\%). The final solution was maintained under magnetic agitation for $1 \mathrm{~h}$ at room temperature. After the reaction, the sample was washed several times and then air dried.

The alloy containing Pd and 3 wt \% of Ce was melted in an arc-furnace under inert atmosphere. The alloy was then cold-rolled up to $200 \mu \mathrm{m}$ of thickness and $15 \mathrm{~mm} \times 300 \mathrm{~mm}$ ribbon plates were cut. These were subjected to thermal treatment at $800{ }^{\circ} \mathrm{C}$ for $24 \mathrm{~h}$ to promote oxygen diffusion in the Pd matrix and the formation of Ce-oxides. The oxide grows in needle or plates shapes, in accordance with the [110] direction of Pd, due to the perfect accommodation between the lattice parameters of $\mathrm{CeO}_{2}(5.42 \AA)$ and the diagonal of the Pd cubic cell (5.50 $\AA$ ) in the (110) plane, as shown in Fig. 7. After the oxidation step, the $\mathrm{PdCeO}_{2}$ alloy was hydrogenated 


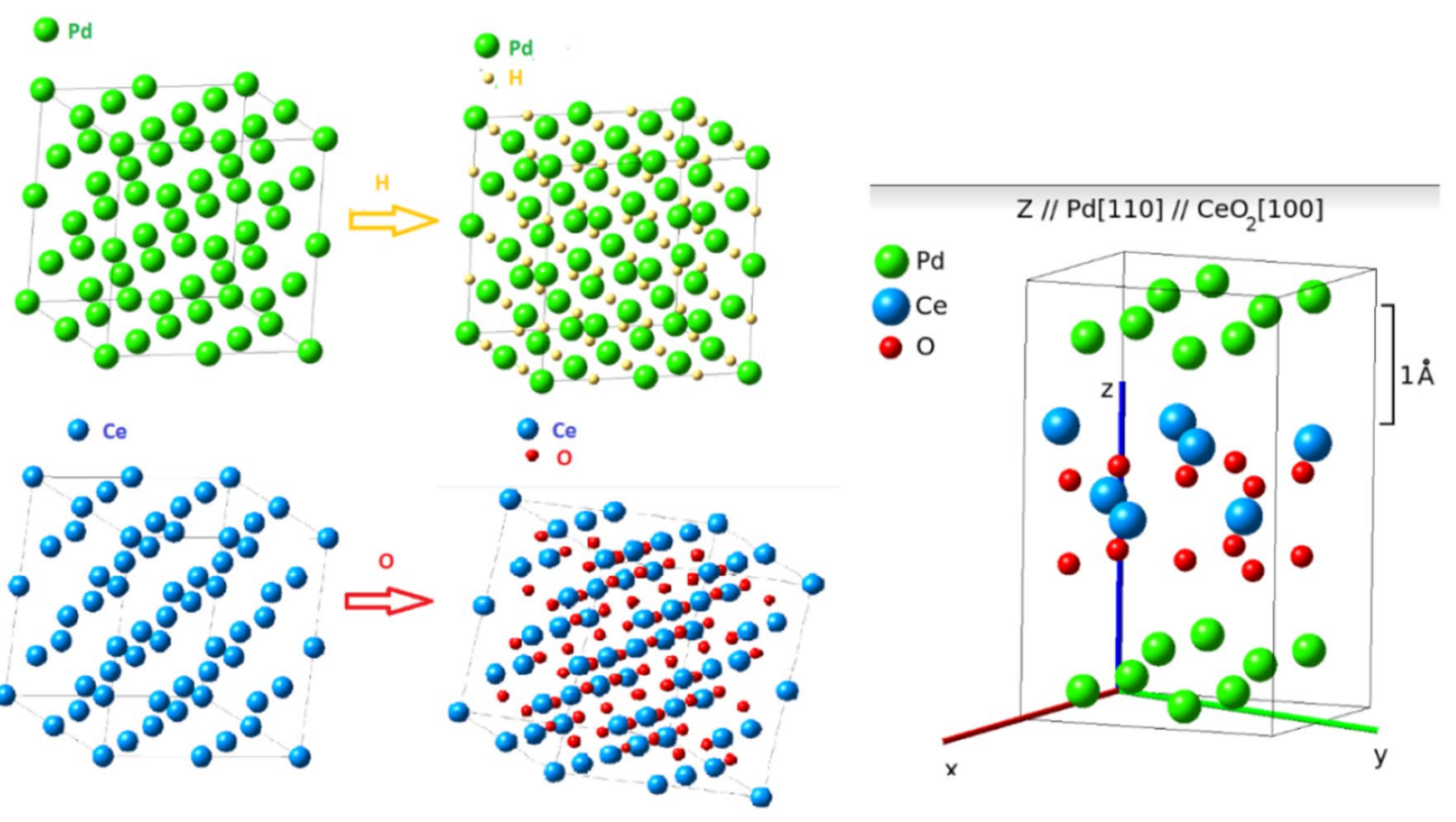

Figure 7. Crystalline cells of $\mathrm{Pd}$ and $\mathrm{Pd}$-hydride, $\mathrm{CeO}_{2}, \mathrm{PdCeO}_{2}$ and $\mathrm{Pd}-\mathrm{CeO}_{2}$ Interface obtained from $\mathrm{MEDEA}$ simulation software.

and submitted to mechanical milling using a ball mill at $300 \mathrm{rpm}$ for $12 \mathrm{~h}$. Hydrogenation was performed to facilitate the comminution of the alloy. The hydrogen absorbed by the alloy was then desorbed during milling, due to the heating caused by friction between the balls of the mill.

An ultrasonic probe was used for dispersion of the $\mathrm{PdNC}$ and $\mathrm{PdCeO}_{2}$ nanoparticles in distilled water for $1 \mathrm{~min}$. PdNC nanofluids with concentrations of $0.2 \mathrm{~g} / \mathrm{L}$ and $0.4 \mathrm{~g} / \mathrm{L}$, as well as a $\mathrm{PdCeO}_{2}$ nanofluid with concentration of $0.4 \mathrm{~g} / \mathrm{L}$, were prepared. Half of the produced nanofluid samples were hydrogenated to form nanoparticles of metal hydride. These nanofluids were bubbled with hydrogen gas at the pressure of 2 bar and constant flow for $1 \mathrm{~h}$ at room temperature.

Both nanocrystalline materials were characterized by $\mathrm{X}$-ray diffraction using the $\mathrm{Cu}-\mathrm{K}_{\alpha}$ radiation with wavelength of $\lambda=1.5406 \AA$. SEM analyses for morphological characterization of the nanoparticles were conducted with a SEM-FEG equipped with a FEI QUANTA detector FEG 250. TEM analyses with a FEI TECNAI G2F20 HRTEM and a TITAN G2 80-200a were also performed to determine the morphology of the materials. Identification of the chemical composition of the materials was performed via EDS analysis of fields with a TITAN G2 80-200a.

Tests were performed in order to measure the temperature variation in each nanofluid and in distilled water, under the irradiation of a diode-laser. The fluids were pipetted into one well (diameter of $6.75 \mathrm{~mm}$ and height of $11.1 \mathrm{~mm}$ ) of 96 -well plates, with volume of $260 \mu \mathrm{L}$. Heat was provided during $180 \mathrm{~s}$ by a diode-laser (NEWPORT, model 525B at a wavelength of $829.1 \mathrm{~nm}$ ), with collimated beam (Collimator THORLABS CFC-2X-B) and for three different powers ( $154 \mathrm{~mW}, 218 \mathrm{~mW}$ and $439 \mathrm{~mW}$ ). The laser power was measured with an optical meter (THORLABS PM100D with Standard Photodiode Power sensor S121C) and the laser beam exhibited a Gaussian profile with $3.1 \mathrm{~mm}$ of diameter (THORLABS-Compact USB 2.9 CMOS Camera). The collimator was perpendicular to the 96-well plate, coaxial with the well containing the fluid and at a distance of $135 \mathrm{~mm}$ from the fluid surface. Temperature measurements of the surface of the fluids were taken with an infrared camera (FLIR A325), with a frequency of one measurement per second and pixel size of $0.24 \mathrm{~mm}$. Figure 8a shows the apparatus used for the photothermal measurements, while Fig. $8 \mathrm{~b}$ presents a thermal image during the laser irradiation of the well with the nanofluid. Other neighboring wells in the 96-well plate containing distilled water, which were not heated by the diode-laser but served as reference for the temperature measurements, also appear in the figure. The heating experiments were carried out in triplicates to ensure reproducibility. The curves presented for the temperature variations correspond to the mean values of the three experiments, which were conducted in a room with controlled ambient temperature $\left(23.5 \pm 0.5^{\circ} \mathrm{C}\right)$.

The measured temperatures were also used for the calculation of the photothermal conversion efficiencies of the produced nanofluids, by following a procedure similar to that proposed by Roper et al. ${ }^{35}$. While these authors used the transient temperature variation during the cooling period and the steady-state temperature after the heating period to calculate the photothermal conversion efficiency, in this work we used an inverse analysis with the transient temperature measurements during the heating period. The model parameters were estimated with the temperature measurements in experiments with distilled water and with the produced nanofluids. The inverse parameter estimation problems were solved within the Bayesian framework of statistics by using the Markov Chain Monte Carlo (MCMC) method $^{36}$. This method allows for the estimation of mean values and the related uncertainties of the model parameters, through stochastic simulation of the posterior probability distribution function. The values reported in this work for the photothermal conversion efficiencies are the means of the 

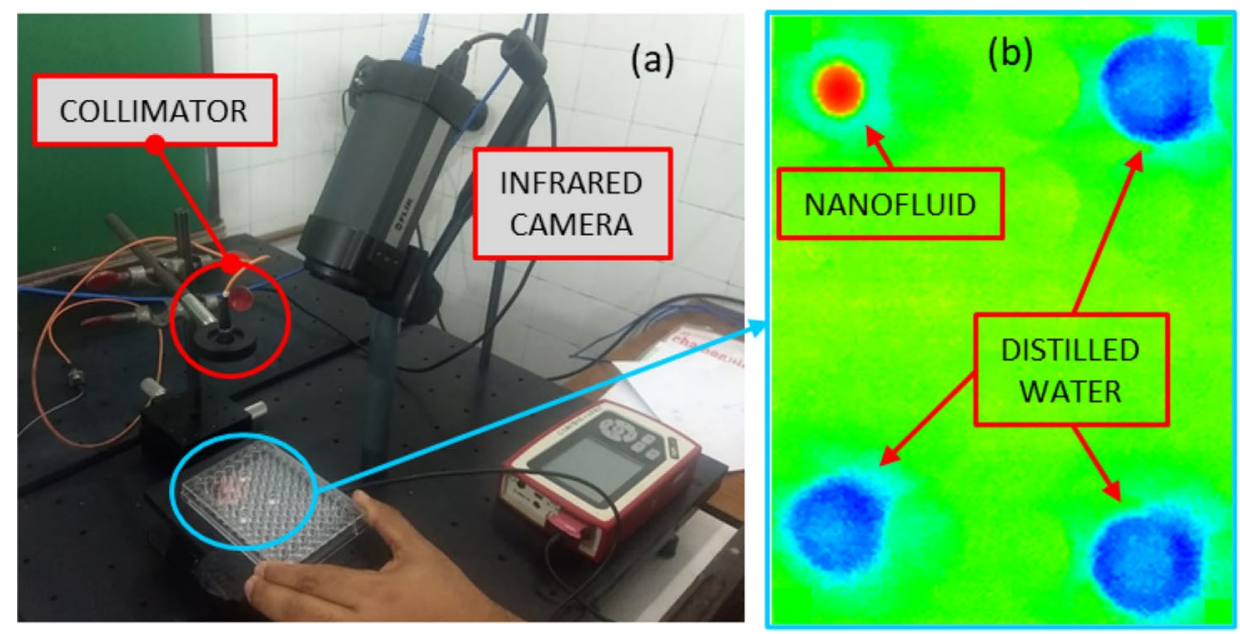

Figure 8. (a) Apparatus for photothermal analysis and (b) typical thermal image during the heating of the nanofluid.

equilibrium distributions obtained with the Markov chains. The reported standard deviations of the photothermal conversion efficiencies were obtained from the standard deviations of the samples in the equilibrium Markov chains and by applying uncertainty propagation.

\section{Conclusions}

Palladium nanocubes were synthesized via chemical precipitation in the presence of CTAB as a surfactant. The synthesis method was effective, resulting in nanocubes free of contamination and about $20 \mathrm{~nm}$ in size. Internal oxidation and mechanical grinding were also used to produce a nanostructured $\mathrm{PdCeO}_{2}$ alloy, which presented the formation of nano-Ceria in the palladium matrix. The materials were tested in experiments involving the heating of nanofluids with a diode-laser. Temperature variations of the nanofluids were substantially larger than that of distilled water, demonstrating the photothermal effect of the nanoparticles developed in this work, including those that were hydrogenated. Our results reveal a great potential for the application of these nanoparticles in the hyperthermia cancer therapy.

Received: 25 March 2020; Accepted: 14 September 2020

Published online: 16 October 2020

\section{References}

1. Wang, F. et al. Bio-mimetic nanostructure self-assembled from Au@Ag heterogeneous nanorods and phage fusion proteins for targeted tumor optical detection and photothermal therapy. Sci. Rep. 4, 6808. https://doi.org/10.1038/srep06808 (2015).

2. Ahmed, K., Tabuchi, Y. \& Kondo, T. Hyperthermia: an effective strategy to induce apoptosis in cancer cells. Apoptosis 20, 14111419. https://doi.org/10.1007/s10495-015-1168-3 (2015).

3. Zhao, P. et al. Local generation of hydrogen for enhanced photothermal therapy. Nat. Commun. 9, 4241. https://doi.org/10.1038/ s41467-018-06630-2 (2018).

4. Ohsawa, I. et al. Hydrogen acts as a therapeutic antioxidant by selectively reducing cytotoxic oxygen radicals. Nat. Med. 13, 688-694. https://doi.org/10.1038/nm1577 (2007).

5. Chen, M. et al. Safety profile of two dimensional Pd nanosheets for photothermal therapy and photoacoustic imaging. Nano Res. 10, 1234-1248. https://doi.org/10.1007/s12274-016-1349-6 (2017).

6. Li, W. et al. In vivo photoacoustic imaging of brain injury and rehabilitation by high-efficient NIR dye labeled mesenchymal stem cells with enhanced brain barrier permeability. Adv. Sci. https://doi.org/10.1002/advs.201700277 (2018).

7. Yan, W. et al. Oxygen reduction reaction and hydrogen evolution reaction catalyzed by carbon-supported molybdenum-coated palladium nanocubes. Int. J. Hydrog. Energy 43, 17132-17141. https://doi.org/10.1016/j.ijhydene.2018.07.097 (2018).

8. Woo, J.-A. et al. Fast response of hydrogen sensor using palladium nanocube- $\mathrm{TiO}_{2}$ nanofiber composites. Int. J. Hydrog. Energy 42, 18754-18761. https://doi.org/10.1016/j.ijhydene.2017.04.189 (2017).

9. Lee, C.-L., Chiou, H.-P. \& Liu, C.-R. Palladium nanocubes enclosed by (100) planes as electrocatalyst for alkaline oxygen electroreduction. Int. J. Hydrog. Energy 37, 3993-3997. https://doi.org/10.1016/j.ijhydene.2011.11.118 (2012).

10. Sun, X. et al. Long-term atomistic simulation of hydrogen absorption in palladium nanocubes using a diffusive molecular dynamics method. Int. J. Hydrog. Energy 43, 5657-5667. https://doi.org/10.1016/j.ijhydene.2018.01.169 (2018).

11. Chong, Y. et al. Palladium concave nanocrystals with high-index facets accelerate ascorbate oxidation in cancer treatment. Nat. Commun. 5, 4861. https://doi.org/10.1038/s41467-018-07257-z (2018).

12. Xie, X. et al. Large-scale synthesis of palladium concave nanocubes with high-index facets for sustainable enhanced catalytic performance. Sci. Rep. 5, 8515. https://doi.org/10.1038/srep08515 (2015).

13. Li, G. et al. Hydrogen storage in Pd nanocrystals covered with a metal-organic framework. Nat. Mater. 13, 802-806. https://doi. org/10.1038/nmat4030 (2014)

14. Gnad, C. et al. Synthesis of methyl palladium complexes on silica as single site catalysts activating C-Cl bonds in heck reactions. J. Catal. 375, 257-266. https://doi.org/10.1016/j.jcat.2019.06.004 (2019).

15. Chen, S. et al. Palladium catalyzed cross-dimerization of terminal acetylenes and acrylates. Tetrahedron Lett. 60, 1234-1237. https ://doi.org/10.1016/j.tetlet.2019.03.062 (2019). 
16. Pourreza, N. \& Abdollahzadeh, R. Colorimetric sensing of palladium ions based on in situ generation of palladium nanoparticles as an activator for the thionine-hydrazine reaction. Talanta 196, 211-216. https://doi.org/10.1016/j.talanta.2018.12.050 (2019).

17. Liu, Q. et al. Two operating modes of palladium film hydrogen sensor based on suspended micro hotplate. Int. J. Hydrog. Energy 44, 11259-11265. https://doi.org/10.1016/j.ijhydene.2019.02.228 (2019).

18. Zhang, L. et al. Novel approach for recovery of palladium in spent catalyst from automobile by a capture technology of eutectic copper. J. Clean. Prod. 239, 118093. https://doi.org/10.1016/j.jclepro.2019.118093 (2019).

19. Bakhshi, F. \& Farhadian, N. Improvement of hydrogen storage capacity on the palladium-decorated N-doped graphene sheets as a novel adsorbent: a hybrid MD-GCMC simulation study. Int. J. Hydrog. Energy 44, 13655-13665. https://doi.org/10.1016/j.ijhyd ene.2019.04.005 (2019).

20. Zhao, J. et al. Effect of palladium addition on electrochemical hydrogen storage properties of $\mathrm{Co}_{0.9} \mathrm{Cu}_{0.1}$ Si alloy. Solid State Sci. 91, 1-6. https://doi.org/10.1016/j.solidstatesciences.2019.02.011 (2019).

21. Adams, B. D. \& Chen, A. The role of palladium in a hydrogen economy. Mater. Today 14, 282-289. https://doi.org/10.1016/S1369 $-7021(11) 70143-2(2011)$.

22. Santos, D. S. et al. Hydrogen diffusivity and solubility in palladium alloys. J. Alloys Compd. 356-357, 236-239. https://doi. org/10.1016/S0925-8388(03)00245-7 (2003).

23. Züchner, H. \& Rauf, T. Electrochemical isotherm measurements on the Pd-H and PdAg-H systems. J. Less Common Met. 172174(Part B), 816-823. https://doi.org/10.1016/0022-5088(91)90208-L (1991).

24. Semaltianos, N. G. et al. Palladium or palladium hydride nanoparticles synthesized by laser ablation of a bulk palladium target in liquids. J. Colloid Interface Sci. 402, 307-311. https://doi.org/10.1016/j.jcis.2013.03.062 (2013).

25. Xu, C. \& Qu, X. Cerium oxide nanoparticle: a remarkably versatile rare earth nanomaterial for biological applications. NPG Asia Mater. 6, e90. https://doi.org/10.1038/am.2013.88 (2014).

26. Eriksson, P. et al. Cerium oxide nanoparticles with antioxidant capabilities and gadolinium integration for MRI contrast enhancement. Sci. Rep. 8, 6999. https://doi.org/10.1038/s41598-018-25390-z (2018).

27. Azambuja, V. M. et al. Study of an internally-oxidized $\mathrm{Pd}_{0.97} \mathrm{Ce}_{0.03}$ alloy. Scr. Mater. 54, 1779-1783. https://doi.org/10.1016/j.scrip tamat.2006.01.039 (2006).

28. Miller, M. et al. Nano-palladium is a cellular catalyst for in vivo chemistry. Nat. Commun. 8, 15906. https://doi.org/10.1038/ncomm s15906 (2017).

29. Deutges, M. et al. Hydrogen diffusivities as a measure of relative dislocation densities in palladium and increase of the density by plastic deformation in the presence of dissolved hydrogen. Acta Mater. 82, 266-274. https://doi.org/10.1016/j.actamat.2014.09.013 (2015).

30. Xiong, Y. et al. Synthesis and mechanistic study of palladium nanobars and nanorods. J. Am. Chem. Soc. 129, 3665-3675. https:// doi.org/10.1021/ja0688023 (2007).

31. Fan, F.-R. et al. An effective strategy for room-temperature synthesis of single-crystalline palladium nanocubes and nanodendrites in aqueous solution. Cryst. Growth Des. 9, 2335-2340. https://doi.org/10.1021/cg801231p (2009).

32. Das, M. et al. Auto-catalytic ceria nanoparticles offer neuroprotection to adult rat spinal cord neurons. Biomaterials 28, $1918-1925$. https://doi.org/10.1016/j.biomaterials.2006.11.036 (2007).

33. Fukai, Y. The Metal-Hydrogen System. Springer Series in Materials Science 2nd edn (Springer, Berlin, 2005).

34. Azambuja, V. M. \& dos Santos, D. S. Hydrogen diffusivity and solubility in a Pd-Al alloy under conditions of hydride formation. J. Alloys Compd. 383, 219-223. https://doi.org/10.1016/j.jallcom.2004.04.064 (2004).

35. Roper, D., Ahn, W. \& Hoepfner, M. Microscale heat transfer transduced by surface plasmon resonant gold nanoparticles. J. Phys. Chem. C 111, 3636-3641. https://doi.org/10.1021/jp064341w (2007).

36. Orlande, H. et al. (eds) Thermal Measurements and Inverse Techniques (CRC Press, Boca Raton, 2011).

\title{
Acknowledgements
}

Authors would like to acknowledge CNPq, CAPES, FAPEAM and FAPERJ for the financial support of this work.

\section{Author contributions}

The project was supervised by D.S.S. and H.R.B.O. The synthesis of the alloys and experiments were performed by C.C.R.C and V.A.F.N; N.P.S. and A. V. C. The laser experiments was adjusted by C.L.C. The data was analyzed, discussed and the manuscript was prepared by all authors.

\section{Competing interests}

The authors declare no competing interests.

\section{Additional information}

Correspondence and requests for materials should be addressed to D.S.S.

Reprints and permissions information is available at www.nature.com/reprints.

Publisher's note Springer Nature remains neutral with regard to jurisdictional claims in published maps and institutional affiliations.

\begin{abstract}
Open Access This article is licensed under a Creative Commons Attribution 4.0 International License, which permits use, sharing, adaptation, distribution and reproduction in any medium or format, as long as you give appropriate credit to the original author(s) and the source, provide a link to the Creative Commons licence, and indicate if changes were made. The images or other third party material in this article are included in the article's Creative Commons licence, unless indicated otherwise in a credit line to the material. If material is not included in the article's Creative Commons licence and your intended use is not permitted by statutory regulation or exceeds the permitted use, you will need to obtain permission directly from the copyright holder. To view a copy of this licence, visit http://creativecommons.org/licenses/by/4.0/.
\end{abstract}

(C) The Author(s) 2020 